\title{
Long axis electromechanics during dobutamine stress in patients with coronary artery disease and left ventricular dysfunction
}

\author{
A M Duncan, C A O'Sullivan, G S Carr-White, D G Gibson, M Y Henein
}

\begin{abstract}
Objective-To dissociate the effect of inotropy from activation change during dobutamine stress on left ventricular long axis function in patients with coronary artery disease (CAD).

Methods-25 patients with CAD and normal left ventricular cavity size and 30 with cavity dilatation-18 with normal activation (DCM-NA) and 12 with left bundle branch block (DCMLBBB) - were compared with 20 controls. 12 lead ECG and septal long axis echograms were assessed at rest and peak dobutamine stress. Amplitude, shortening and lengthening velocities, postejection shortening, $\mathrm{Q}$ wave to onset of shortening (Q-OS), and A2 to onset of lengthening (A2-OL) were measured. Inotropy was evaluated from peak aortic acceleration.

Results-In controls, amplitude, shortening and lengthening velocities, and peak aortic acceleration increased with stress; QRS, Q-OS, and A2-OL shortened (all p < 0.001); and contraction remained coordinate. In the group of patients with CAD and normal left ventricular cavity size, shortening velocity and peak aortic acceleration increased with stress $(p<0.005)$. However, amplitude and lengthening velocity did not change, QRS, Q-OS, and A2-OL lengthened $(\mathrm{p}<0.01)$, and incoordination appeared. Results were similar in the group with DCM-NA. In the DCM-LBBB group, shortening velocity and peak aortic acceleration increased modestly with stress $(\mathrm{p}<0.01)$ but amplitude, lengthening velocity, QRS, Q-OS, A2-OL, and incoordination remained unchanged. Overall, change in shortening velocity correlated with that in peak aortic acceleration $\left(r^{2}=0.71\right)$, in amplitude with that in lengthening velocity $\left(r^{2}=0.74\right)$, and in QRS with both Q-OS $\left(r^{2}=0.69\right)$ and A2-OL $\left(r^{2}=0.63\right)$.

Conclusion-The normal long axis response to dobutamine reflects both inotropy and rapid activation. In CAD, inotropy is preserved with development of ischaemia but the normal increase in amplitude is lost and prolonged activation delays the time course of shortening, causing pronounced incoordination. Overall, shortening rate uniformly reflects inotropy while lengthening rate depends mainly on systolic amplitude rather than primary diastolic involvement, even with overt ischaemia.

(Heart 2001;86:397-404)
\end{abstract}

Keywords: stress echocardiography; activation; inotropy; incoordination

Normal left ventricular contraction depends on the coordinate function of longitudinally and circumferentially directed muscle fibres. ${ }^{12}$ While the major component of myocardial mass is arranged circumferentially, interference with the extent of timing of longitudinal fibres causes ventricular contraction to become incoordinate, particularly during the isovolumic periods. ${ }^{3}$ Longitudinal fibres are mainly subendocardial $^{4}$ and are thus disproportionately sensitive to ischaemia, which, at rest, reduces the amplitude of contraction and delays its time course..$^{56}$ In addition, the timing of long axis contraction is sensitive to changes in activation. ${ }^{7}$ In normal subjects, dobutamine stress causes QRS duration to shorten, with a related fall in isovolumic periods, while in patients with coronary artery disease (CAD) exactly the reverse changes occur. ${ }^{9}$ These effects are independent of the positive inotropic action of the drug. It was the aim of the present study to investigate the effects of dobutamine stress on long axis mechanical function in normal subjects and in patients with CAD and ventricular involvement of varying severity. In particular, we wished to investigate interactions between altered activation, ischaemia, and the positive inotropic effect of the drug, with a view to analysing disturbances of long axis function that may occur in disease.

\section{Methods}

Fifty five patients with CAD were studied (table 1 shows clinical details). Left ventricular cavity size was normal in 25 patients and dilated in 30 (minor axis dimensions both greater than the upper $95 \%$ confidence limit of normal: end diastolic dimension $>5.6 \mathrm{~cm}$ and end systolic dimension $>4.0 \mathrm{~cm}$ ). Eighteen of the 30 patients with left ventricular cavity dilatation had normal activation with a QRS duration of $>120 \mathrm{~ms}$ (DCM-NA) and 12 had left bundle branch block (DCM-LBBB). All 30 had a restrictive filling pattern with a combination of a short isovolumic relaxation time ( $<40 \mathrm{~ms}$, the lower $95 \%$ confidence limit of normal), dominant left ventricular early diastolic filling, and an $\mathrm{E}$ wave deceleration time of $<120 \mathrm{~ms}^{10}{ }^{11}$ Cardiac catheterisation, showing at least two vessel disease including $>70 \%$ stenosis of the left anterior descending artery, had been performed within the previous 15 months in all patients with cavity dilatation. Left anterior descending artery disease was found on 
Table 1 Clinical details

\begin{tabular}{|c|c|c|c|}
\hline Variable & $\begin{array}{l}\text { Controls } \\
(n=20)\end{array}$ & $\begin{array}{l}\text { Normal LV cavity } \\
(n=25)\end{array}$ & $\begin{array}{l}\text { Dilated LV cavity and } \\
\text { restrictive filling }(n=30)\end{array}$ \\
\hline Age (years) & $58 \quad(11)$ & $63(10)$ & 63 (9) \\
\hline Male:female & $5: 12$ & $16: 9$ & $27: 3$ \\
\hline $\operatorname{EDD}(\mathrm{cm})$ & $5.0(0.5)$ & $4.9(0.5)$ & $6.9(0.8)^{\star}$ \\
\hline $\operatorname{ESD}(\mathrm{cm})$ & $3.4(0.5)$ & $3.3(0.5)$ & $5.9(0.9)^{\star}$ \\
\hline SF (\%) & $34 \quad(8)$ & $32(8)$ & $17(5)^{\star}$ \\
\hline IVRT (ms) & 59 (11) & 62 (16) & $(14)^{\star}$ \\
\hline $\mathrm{E}$ decel (ms) & $148 \quad(15)$ & $144 \quad(20)$ & $79(19)^{\star}$ \\
\hline \multicolumn{4}{|l|}{ Clinical history } \\
\hline Myocardial infarction & & 8 & 16 \\
\hline Diabetes & & 4 & 3 \\
\hline Hypertension & & 5 & 7 \\
\hline \multicolumn{4}{|l|}{ Medication } \\
\hline Diuretics & & 0 & 24 \\
\hline Nitrates & & 10 & 13 \\
\hline$\beta$ Blocker & & 9 & 10 \\
\hline ACE inhibitor & & 12 & 26 \\
\hline
\end{tabular}

Values are mean (SD).

${ }^{\star} \mathrm{p}<0.001$ versus control resting value.

$\mathrm{ACE}$, angiotensin converting enzyme; E decel, E wave deceleration time; EDD, end diastolic dimension; ESD, end systolic dimension; IVRT, isovolumic relaxation time; LV, left ventricular; $\mathrm{SF}$, shortening fraction
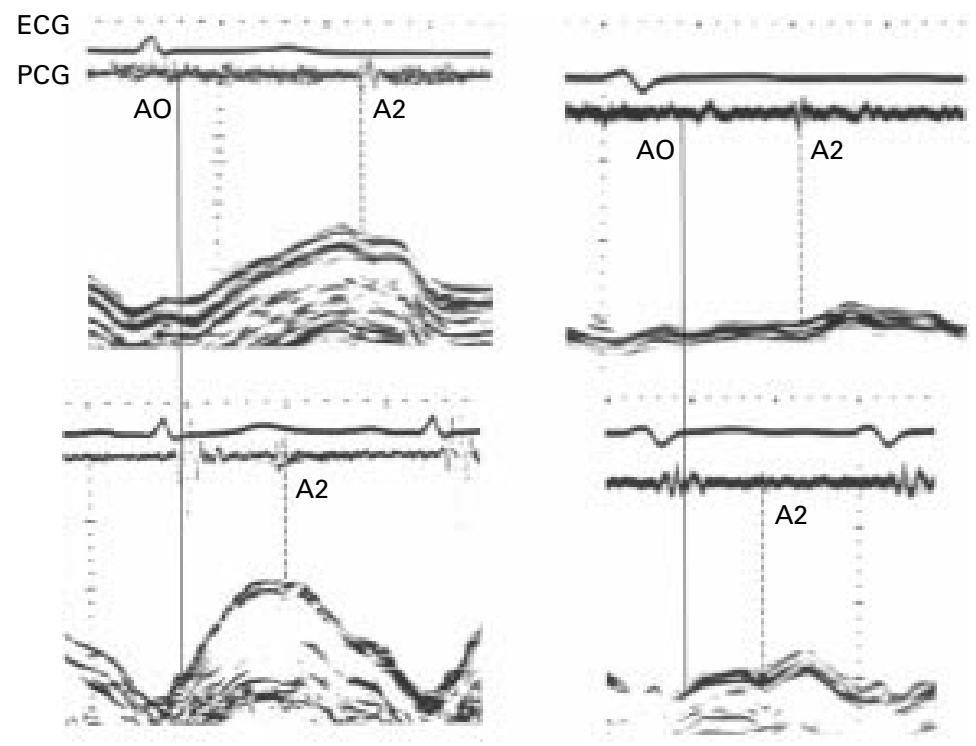

Figure 1 Left: long axis shortening during ejection normally increases during stress. In patients with dilated cardiomyopathy and normal activation (right), shortening during ejection, already lower than normal at rest, falls further during stress. A2, aortic valve closing; AO, aortic valve opening; PCG, phonocardiogram.

angiography in 16 patients with normal left ventricular cavity size, with the remainder of patients showing anterior ECG changes during treadmill testing. No patient had experienced an acute ischaemic event in the seven days before the study. Patients with structural valve disease or arrhythmia at rest were excluded. The two patient groups were compared with each other and with 20 control subjects of a similar age, none of whom had a history of angina, hypertension, or diabetes. The Royal Brompton and Harefield ethics committee approved the study protocol and all subjects gave written informed consent.

RESTING DOPPLER ECHOCARDIOGRAPHY

Transthoracic echocardiography was performed using a Hewlett Packard Sonos 5500 echocardiograph (Andover, Massachusetts, USA) and a $2.5 \mathrm{MHz}$ transducer, with the operator blinded to the results of concurrent diagnostic tests for CAD. With the subject in the left semilateral position, cross sectional two dimensional guided $\mathrm{M}$ mode recordings of the left ventricular minor axis were made in the standard left parasternal long axis view with the cursor at the tips of the mitral valve leaflets. Left ventricular long axis recordings were obtained in the apical four chamber view with the $M$ mode cursor positioned at the left and septal angles of the mitral ring. ${ }^{12}{ }^{13}$ The left ventricular posterior long axis was recorded from the apical two chamber view at the posterior angle of the mitral ring. All long axis $M$ mode traces were later digitised off line. ${ }^{14}$ Transmitral flow velocities were obtained from the apical four chamber view using pulsed wave Doppler echocardiography and aortic flow from the apical five chamber view, with the sample volume at the tips of the respective valve leaflets. Recordings were obtained at a paper speed of $100 \mathrm{~mm} / \mathrm{s}$, with an ECG (lead II) and a phonocardiogram superimposed (fig 1).

DOBUTAMINE STRESS PROTOCOL

Dobutamine was administered through an infusion pump (IVAC 770 syringe driver, Alaris Medical System, Hampshire, UK) starting at a rate of $5 \mu \mathrm{g} / \mathrm{kg} / \mathrm{min}$, increased every three minutes by a similar amount to a maximum dose of $40 \mu \mathrm{g} / \mathrm{kg} / \mathrm{min}$. Additional bolus doses of atropine $(300 \mu \mathrm{g})$ were added to augment heart rate in patients who did not reach predetermined stress end points by the end of stage 8 of the dobutamine protocol. In the control group, stress end points were taken as reaching $85 \%$ predicted target heart rate (220 minus age in years (beats/min)) or the maximum dobutamine dose. In patients, the stress end points were the onset of chest pain or breathlessness, ST segment shift (elevation or depression $>2 \mathrm{~mm}$ ), arrhythmia (including frequent ventricular extrasystoles), or a $20 \mathrm{~mm} \mathrm{Hg}$ drop in arterial pressure. Long axis echocardiograms and Doppler aortic and mitral flow velocities were repeated at peak stress, all with an ECG and phonocardiogram superimposed. Systolic and diastolic blood pressures were recorded at each stage using a Critikon Dinamap monitor (Critikon Inc, Tampa, Florida, USA).

\section{STRESS ECHOCARDIOGRAPHIC MEASUREMENTS} ECG

A standard 12 lead ECG was recorded at rest and at each stage of dobutamine stress by a Hewlett-Packard Pagewriter Xli with built in analysis package. ECG intervals were measured automatically and registered on a printed chart at a speed of $25 \mathrm{~mm} / \mathrm{s}$. The frequency response of the machine was 0.05$150 \mathrm{~Hz}$ with the baseline filter $(0.4 \mathrm{~Hz})$ inactivated. Heart rate and QRS duration were determined directly using in built software. ST segment elevation or depression was measured manually $80 \mathrm{~ms}$ after the J point of the ST segment in the lead showing the most change. A shift of $2 \mathrm{~mm}$ or more was taken to indicate significant inducible ischaemia. LBBB was diagnosed on the basis of a QRS duration $>120 \mathrm{~ms}$, absent $\mathrm{Q}$ waves, wide slurred $\mathrm{R}$ 
Table 2 Regression analysis and correlation between $Q R S$ and $Q$ wave to onset of shortening (Q-OS) or $A 2$ to onset of lengthening ( $A 2-O L)$

\begin{tabular}{lllll}
\hline Variable & Controls & Normal LV cavity & DCM-NA & DCM-LBBB \\
\hline q-OS & & & & \\
$\quad$ Intercept (ms) & $-32.9(10.9)$ & $-1.1(8.1)^{\star \star}$ & $+9.7(10.6) \dagger$ & $-13.3(10.4) \Omega S$ \\
$\quad$ Slope & $3.2(2.2)$ & $1.3(0.9)^{\star}$ & $1.8(1.3)$ & $1.6(1.0)$ \\
$\quad$ Correlation coefficient $\left(r^{2}\right)$ & 0.71 & 0.68 & 0.70 & 0.69 \\
A2-OL & & & & \\
$\quad$ Intercept (ms) & $-12.3(11.0)$ & $+6.9(8.9)^{\star \star}$ & $+11.1(11.8)$ & $-7.3(20.6) \subseteq$ \\
$\quad$ Slope & $3.0(2.3)$ & $1.1(1.1)^{\star}$ & $1.5(1.4)$ & $1.9(1.8)$ \\
$\quad$ Correlation coefficient $\left(r^{2}\right)$ & 0.65 & 0.62 & 0.63 & 0.63 \\
\hline
\end{tabular}

Values are mean (SD).

${ }^{\star} \mathrm{p}<0.005,{ }^{\star \star} \mathrm{p}<0.001$ normal LV cavity versus controls; $\mathrm{tp}<0.001$ patients with LV cavity dilatation with normal activation (DCM-NA) versus normal LV cavity; $\$ p<0.01$, \\p $<0.001$ patients with LV cavity dilatation with left bundle branch block (DCM-LBBB) versus DCM-NA; $\lceil\mathrm{p}<0.01$ significantly different from zero.

waves in V5 and V6, and monophasic QS or RS waves in V1 and V2.

\section{Echocardiogram}

Left ventricular minor axis dimensions were taken at end diastole (the onset of the QRS complex) and at end systole (at the first high frequency vibration of the aortic component of the second heart sound on the phonocardiogram, A2). Since splitting of the second sound may be reversed in patients with LBBB, A2 was identified from the sound synchronous with the onset of the closure artefact on the aortic Doppler record. Isovolumic relaxation time was measured from A2 to mitral cusp separation on the $M$ mode trace of the mitral echogram and mitral $\mathrm{E}$ wave deceleration time (from the peak of the $\mathrm{E}$ wave to the termination of early diastolic flow) was obtained from the mitral Doppler trace. Ejection time was measured as the interval between the onset of forward flow pulse across the aortic valve to the onset of the aortic closure artefact. Total ventricular systolic long axis excursion was measured as the amplitude of mitral ring motion occurring between the end of the QRS and the peak innermost point (towards the ventricular cavity) at or after A2. The amplitude of postejection shortening was measured as the component of shortening after A2. The coordination of the long axis motion was assessed as the ratio of shortening during ejection to the total amount of systolic shortening (including postejection shortening), expressed as a percentage. The lower this value, the greater the degree of incoordination. Peak shortening and lengthening velocities were measured from the digitised long axis traces. Septal electromechanical delay was measured from the $\mathrm{Q}$ wave of the ECG to the onset of long axis shortening (Q-OS) in patients with normal activation and from the onset of the QRS in patients with LBBB. The duration of postejection shortening was measured from A2 to the onset of lengthening (A2-OL). Aortic velocity traces were digitised off line $(100 \mathrm{~Hz})$ and the peak aortic acceleration rate, expressed in $\mathrm{G}\left(1 \mathrm{G}=9.81 \mathrm{~m} / \mathrm{s}^{2}\right)$, was derived from the first differential of the velocity trace with respect to time. ${ }^{9}$

STATISTICAL ANALYSIS

Values are expressed as mean (SD). Resting values of controls and patients were compared by Student's unpaired $t$ test. Within control and patient groups, rest and stress values were compared by paired $t$ tests. In view of multiple $t$ tests, a significant difference was taken as $\mathrm{p}<0.01$. Correlation was determined by linear regression analysis and the SDs of the intercepts and slopes were determined (table 2).

REPRODUCIBILITY

Echocardiographic findings were analysed by two investigators, both blinded to the original diagnosis and to the other's findings. Duplicate determinations of the timing of shortening and lengthening with respect to the $\mathrm{Q}$ wave and $\mathrm{A} 2$, long axis velocities, and peak aortic acceleration were assessed in 20 patients. Intraobserver coefficient of variability ranged from $3.7-5.6 \%$ and interobserver variability from $4.0-6.5 \%$. The reproducibility of ECG data has been previously reported. ${ }^{9}$

\section{Results}

There was no age difference between patients and the control group (table 1) although there were more women in the latter group. At rest, total long axis amplitude correlated similarly with shortening fraction in individual patients and controls $\left(r^{2}=0.89\right)$ and major regional wall motion abnormalities were not apparent. All those in the control group achieved target heart rate and none developed symptoms or ECG changes. ECG changes were induced in all patients with normal left ventricular size (3 ST elevation, $10 \mathrm{ST}$ depression, and $12 \mathrm{~T}$ wave inversion) and were accompanied by chest pain in 19. All patients with left ventricular cavity dilatation developed symptoms (predominately breathlessness) but ECG changes were less frequent (3 ST elevation, 8 ST depression, and 10 $\mathrm{T}$ wave inversion). Patients in this group were more likely to be taking diuretics and angiotensin converting enzyme inhibitors, although the incidence of $\beta$ blocker treatment was similar irrespective of cavity size. In the interest of simplicity results of septal long axis function alone are presented. They were effectively similar to those of lateral and posterior left ventricular sites.

TOTAL LONG AXIS AMPLITUDE

In patients with CAD and normal left ventricular cavity size, total systolic long axis amplitude at rest was $20 \%$ less than that in the control group $(p<0.01)$, with progressive further depression in those with DCM-NA and DCM-LBBB (table 3). With dobutamine, total systolic amplitude increased by $25 \%$ in the control group $(p<0.001)$, with a concomitant increase in the proportion of overall shortening occurring during ventricular ejection (fig 1). By contrast, the overall amplitude of shortening failed to increase in any patient group and the proportion occurring during ejection fell in all (fig 2).

SHORTENING VELOCITY

At rest, peak long axis shortening velocity fell progressively as left ventricular disease became more severe (table 3). Although it increased with dobutamine in all groups the extent of the increment again fell with increasing severity of 


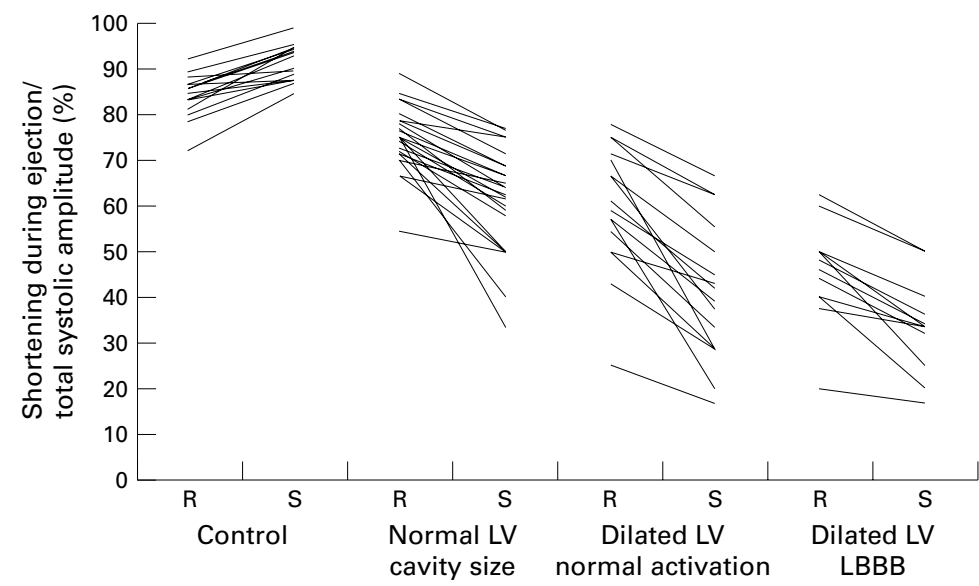

Figure 2 At rest, the percentage of total systolic shortening that occurred during ejection fell as the severity of ventricular disease increased. In the control group, shortening during ejection increased during stress but fell further in all patient groups. $R$, rest; $S$, stress.

left ventricular disease. There was no correlation between the total amplitude of shortening and peak velocity within or between the groups studied.

LENGTHENING VELOCITY

Compared with the control group, resting lengthening velocity was significantly lower in patients with normal left ventricular cavity size, lower still in the DCM-NA group, and less than $5 \%$ normal in patients with DCM-LBBB (table 3). With stress, lengthening velocity increased in the control group but failed to do so in any patient group. There was a single relation between lengthening velocity and total systolic amplitude $\left(r^{2}=0.69\right)$, and between their changes with dobutamine $\left(r^{2}=0.74\right.$, fig $3 \mathrm{~A}$ ), which applied to controls and patients at rest and with stress.

POSTEJECTION SHORTENING

In the control group, postejection shortening was absent at rest and did not appear in any subject during stress (table 3). In eight of the 25 patients with normal left ventricular cavity size, postejection shortening was absent at rest, and Q-OS and A2-OL were both normal. With stress, postejection shortening developed in all eight, and Q-OS and A2-OL was prolonged (from 86 (9) $\mathrm{ms}$ to 106 (15) ms, and from 80 (8) $\mathrm{ms}$ to 100 (8) $\mathrm{ms}$, respectively, both $\mathrm{p}<0.001)$. In the remaining 17 patients postejection shortening was already present at rest and increased with stress (from 1.6
(0.7) $\mathrm{mm}$ to $3.0(0.6) \mathrm{mm}, \mathrm{p}<0.001)$. In them, Q-OS was $50 \mathrm{~ms}$ and $80 \mathrm{~ms}$ longer, and A2-OL was $40 \mathrm{~ms}$ and $70 \mathrm{~ms}$ longer than normal at rest and stress, respectively $(\mathrm{p}<0.001)$. Provided that activation was normal, mean resting postejection shortening amplitude was similar in patients with cavity dilatation to those in whom cavity size was normal and its extent increased similarly with stress. By contrast, postejection shortening amplitude was a little greater (NS) at rest in patients with DCM-LBBB than in those with normal activation and did not change with stress.

\section{TIME INTERVALS}

In the control group, QRS duration, Q-OS, and A2-OL intervals all fell as heart rate increased (table 4). The fall in both Q-OS and A2-OL correlated closely with the reduction in QRS duration $\left(r^{2}=0.71\right.$ and $r^{2}=0.65$, respectively, table 2 , fig 4 ). In patients with normal left ventricular cavity size, resting heart rate and QRS duration were not different from controls but Q-OS and A2-OL were both approximately $30 \mathrm{~ms}$ longer $(\mathrm{p}<0.001)$. With stress, the QRS complex broadened $(\mathrm{p}<0.001)$ and the extent of this broadening correlated with an increase in Q-OS and A2-OL $\left(r^{2}=0.68\right.$ and $r^{2}=0.62$, respectively). The findings were similar in patients with DCM-NA. By contrast, in patients with DCM-LBBB, Q-OS and A2-OL were almost $60 \mathrm{~ms}$ longer $(\mathrm{p}<0.001)$ than normal at rest and twice as long as that in patients with DCM-NA $(p<0.01)$. Despite an increase in heart rate, neither QRS duration nor Q-OS or A2-OL changed with stress. The slopes of these electromechanical relations were similar in all the patient groups (table 2) and all were less than that of the control group. The intercept in control subjects that represented a fall in Q-OS of $33 \mathrm{~ms}$ with zero change in QRS duration was significantly different from zero and larger than in any patient group. Slopes and intercepts for the interval A2-OL were similar between groups.

PEAK AORTIC ACCELERATION

Resting peak aortic acceleration was similar in the control and patient groups with normal left ventricular cavity size but was lower in those in whom the cavity was dilated (table 4). During stress, peak aortic acceleration increased in all groups, although the extent of this increase in absolute terms was less in patients with

Table 3 Septal long axis response to dobutamine stress

\begin{tabular}{|c|c|c|c|c|c|c|c|c|}
\hline \multirow[b]{3}{*}{ Variable } & & & & & \multicolumn{4}{|c|}{ Dilated $L V$ cavity and restrictive filling } \\
\hline & \multicolumn{2}{|c|}{ Controls $(n=20)$} & \multicolumn{2}{|c|}{ Normal LV cavity $(n=25)$} & \multicolumn{2}{|c|}{$Q R S<120 \mathrm{~ms}$} & \multicolumn{2}{|c|}{$Q R S>120 \mathrm{~ms}$} \\
\hline & Rest & Stress & Rest & Stress & Rest & Stress & Rest & Stress \\
\hline Total long axis amplitude $(\mathrm{cm})$ & $1.4(0.3)$ & $1.7(0.2)^{\star \star \star}$ & $1.1(0.3) \dagger$ & $1.1(0.3)$ & $0.8(0.3) \neq \ddagger$ & $0.7(0.3)$ & $0.3(0.2) \S$ & $0.4(0.3)$ \\
\hline Long axis shortening during ejection (\%) & $85(3)$ & $91(6)^{\star \star \star}$ & $75(8)+\dagger$ & $62(13)^{\star \star \star}$ & $61(14) \ddagger \neq \neq$ & $42(16)^{\star \star \star}$ & $46(13) \int$ & $34(12)^{\star \star}$ \\
\hline Shortening velocity $(\mathrm{cm} / \mathrm{s})$ & $6.9(1.6)$ & $11.3(1.7)^{\star \star \star}$ & $4.8(1.9) \dagger \dagger$ & $7.9(3.2)^{\star \star}$ & $2.6(0.8) \neq \neq \ddagger$ & $4.6(2.4)^{\star \star}$ & $1.1(1.2) \rrbracket$ & $2.5(2.4)^{\star}$ \\
\hline Lengthening velocity $(\mathrm{cm} / \mathrm{s})$ & $6.1(1.7)$ & $8.2(2.4)^{\star \star \star}$ & $4.2(2.7)+\dagger$ & $4.6(2.9)$ & $2.2(1.1) \ddagger$ & $2.9(1.8)$ & $0.1(1.8) \S$ & $0.7(2.4)$ \\
\hline Postejection shortening $(\mathrm{mm})$ & 0 & 0 & $0.7(0.9)+\dagger$ & $1.9(1.4)^{\star \star \star}$ & $0.8(1.2)$ & $1.8(1.8)^{\star \star}$ & $1.0(1.2)$ & $1.3(1.5)$ \\
\hline
\end{tabular}

Values are mean (SD).

${ }^{\star} \mathrm{p}<0.01,{ }^{\star \star} \mathrm{p}<0.005,{ }^{\star \star \star} \mathrm{p}<0.001$ stress versus rest within group (paired $\mathrm{t}$ test) $\mathrm{tp}<0.01,+\mathrm{t} \mathrm{p}<0.001$ rest value normal cavity size versus rest value control (unpaired $t$ test); $\neq \mathrm{p}<0.01, \neq \neq \mathrm{p}<0.005, \ddagger \neq \neq \mathrm{p}<0.001$ rest value DCM-NA versus rest value normal cavity (unpaired $t$ test); $\$ \mathrm{p}<0.005$ rest value DCM-LBBB versus rest value DCM-NA (unpaired $t$ test). 

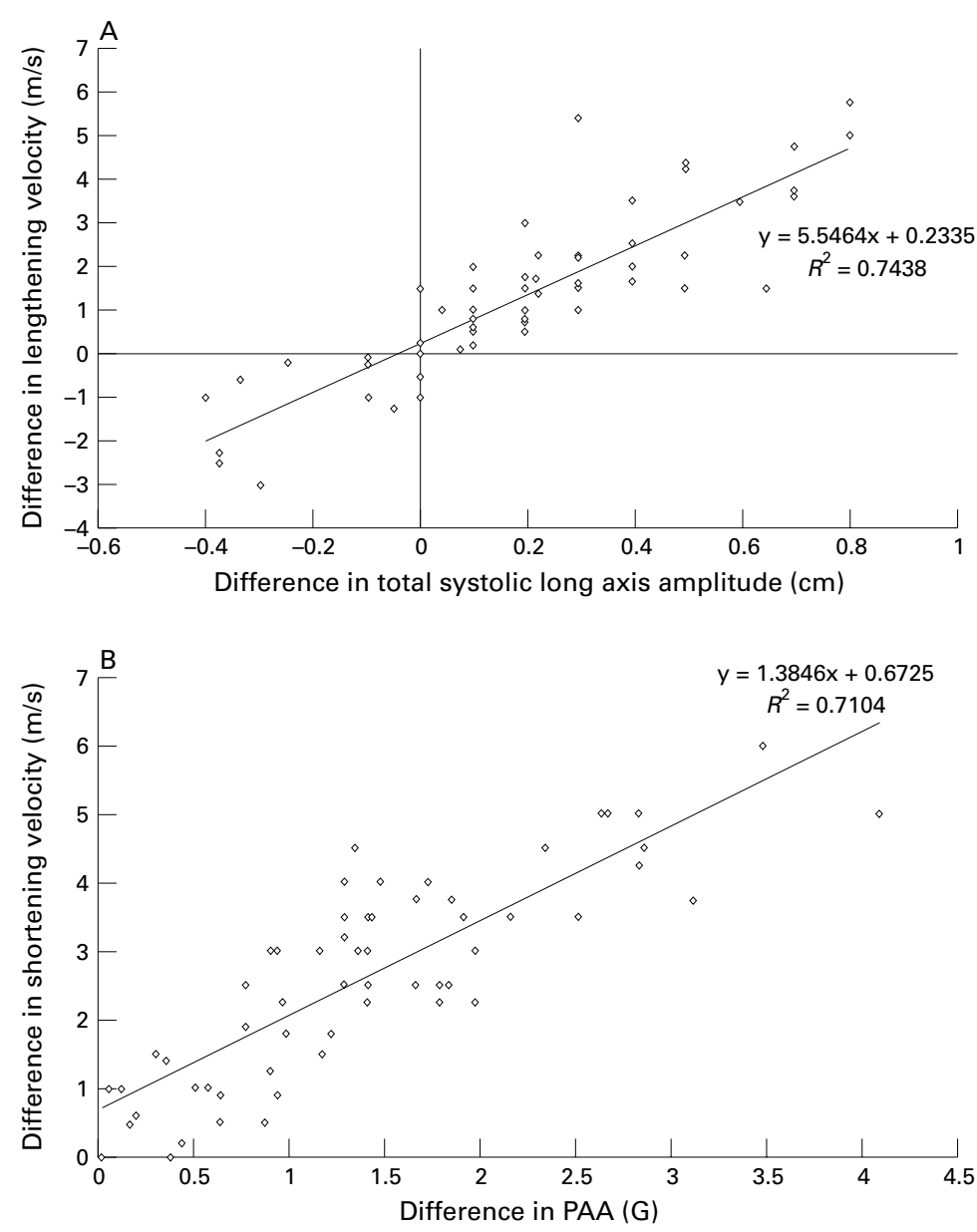

Figure 3 (A) There was a close correlation between the change in total systolic amplitude and lengthening velocity in the control group and all patients when taken as a single group. (B) There was a close correlation between the change in shortening velocity and peak aortic acceleration (PAA) in individual subjects.

DCM-NA and in particular with DCMLBBB. In individual control subjects and patients, there was a single relation between the change in peak aortic acceleration and that in peak long axis shortening velocity $\left(r^{2}=0.71\right.$, fig $\left.3 \mathrm{~B}\right)$.

MITRAL INFLOW VELOCITIES

Peak mitral E wave velocity was similar in control subjects and patients with normal left ventricular cavity size but was greater in those with cavity dilatation and restrictive filling (table 4).
During stress, peak E wave velocity remained unchanged in all groups, although peak A wave velocity increased in patients and control subjects. However, in six patients, all of whom had left ventricular cavity dilatation, the filling pattern became summation in character, with no discernible A wave.

SUBGROUP ANALYSIS

In patients with hypertension, resting echocardiography confirmed left ventricular hypertrophy. Of the five patients with normal left ventricular cavity size and high blood pressure, end diastolic septal thickness was $1.6(0.2) \mathrm{cm}$ compared with $1.0(0.1) \mathrm{cm}$ in the control group, and end diastolic posterior wall thickness was $1.4(0.3) \mathrm{cm}$ compared with 0.9 $(0.2) \mathrm{cm}(\mathrm{p}<0.001)$. Septal thickness was 1.3 $(0.2) \mathrm{cm}$ and posterior wall thickness was 1.3 $(0.2) \mathrm{cm}$ in the seven patients with dilated left ventricular cavity size and hypertension ( $p<0.01$ compared with controls). Neither sex nor resting left ventricular hypertrophy nor diabetes was associated with significant differences in the extent of long axis shortening during ejection, shortening, or lengthening velocities, the amplitude of stress induced postejection shortening, or the timing to onset of long axis shortening or lengthening. Similarly, no differences were seen between patients who were and those who were not taking angiotensin converting enzyme inhibitors or $\beta$ blockers.

\section{Discussion}

The normal left ventricular long axis response to dobutamine infusion proved to be very characteristic. Both the amplitude and peak velocity of motion increased with respect to resting values, although there was no correlation between the two in individual patients. However, the increase in shortening velocity with dobutamine correlated closely with that in peak aortic acceleration, long recognised as a sensitive measure of a positive inotropic stimulus, independent of loading conditions. ${ }^{15}$ This increase in long axis shortening velocity is thus likely to be another direct effect of the positive inotropism of dobutamine. A second effect of dobutamine was that systolic long axis motion started sooner after the $Q$ wave and ended

Table 4 Timing and haemodynamic response to dobutamine stress

\begin{tabular}{|c|c|c|c|c|c|c|c|c|}
\hline \multirow[b]{3}{*}{ Variable } & \multirow{2}{*}{\multicolumn{2}{|c|}{ Controls $(n=20)$}} & \multirow{2}{*}{\multicolumn{2}{|c|}{ Normal LV cavity $(n=25)$}} & \multicolumn{4}{|c|}{ Dilated LV cavity with restrictive filling $(n=30)$} \\
\hline & & & & & \multicolumn{2}{|c|}{$Q R S<120 m s(n=18)$} & \multicolumn{2}{|c|}{$Q R S>120 m s(n=12)$} \\
\hline & Rest & Stress & Rest & Stress & Rest & Stress & Rest & Stress \\
\hline Heart rate (beats/min) & $76(8)$ & $128(9)^{\star \star}$ & $72(13)$ & $126(15)^{\star \star}$ & $70(14)$ & $119(19)^{\star \star}$ & $81(13)$ & $119(12)^{\star \star}$ \\
\hline QRS (ms) & $93(7)$ & $85(9)^{\star \star}$ & $92(13)$ & $105(15)^{\star \star}$ & $95(13)$ & $100(12)^{\star}$ & $151(15) \$ S$ & $153(21)$ \\
\hline $\mathrm{ET}(\mathrm{ms})$ & $270(23)$ & $180(20)^{\star \star}$ & $264(31)$ & $172(20)^{\star \star}$ & $257(44)$ & $201(26)^{\star \star}$ & $209(26) \S$ & $168(21)^{\star \star}$ \\
\hline \multicolumn{9}{|l|}{ Long axis } \\
\hline $\mathrm{q}-\mathrm{OS}(\mathrm{ms})$ & $81(12)$ & $34(5)^{\star \star}$ & $108(23) \dagger$ & $120(17)^{\star}$ & $118(32)$ & $141(43)^{\star \star}$ & $140(32) \rrbracket$ & $146(19)$ \\
\hline $\mathrm{A} 2-\mathrm{OL}(\mathrm{ms})$ & $56(14)$ & $29(9)^{\star \star}$ & 89 (19)† & $101(17)^{\star \star}$ & $83(28)$ & $108(26)^{\star \star}$ & $113(27) \S$ & $114(30)$ \\
\hline \multicolumn{9}{|l|}{ Haemodynamics } \\
\hline $\operatorname{PAA}(\mathrm{G})$ & $1.3(0.3)$ & $3.5(0.5)^{\star \star}$ & $1.3(0.4)$ & $2.9(0.5)^{\star \star}$ & $1.1(0.3)$ & $1.9(0.6)^{\star \star}$ & $1.0(0.2)$ & $1.6(0.6)^{\star}$ \\
\hline Mitral E (m/s) & $0.7(0.2)$ & $0.7(0.2)$ & $0.7(0.2)$ & $0.6(0.2)$ & $0.9(0.2) \ddagger$ & $0.8(0.3)$ & $1.0(0.3)$ & $0.8(0.3)$ \\
\hline Mitral A $(\mathrm{m} / \mathrm{s})$ & $0.7(0.2)$ & $0.9(0.2)^{\star \star}$ & $0.8(0.2)$ & $1.0(0.2)^{\star \star}$ & $0.5(0.2)$ & $0.7(0.3)^{\star \star}$ & $0.4(0.4)$ & $0.5(0.3)^{\star}$ \\
\hline
\end{tabular}

Values are mean (SD).

${ }^{\star} \mathrm{p}<0.01,{ }^{\star \star} \mathrm{p}<0.001$ stress versus rest within group (paired $t$ test); $\mathrm{tp}<0.001$ rest value normal cavity size versus rest value control (unpaired $t$ test); $\ddagger$ : $<<0.001$ rest value DCM-NA versus rest value normal cavity (unpaired $t$ test); $\$ \mathrm{p}<0.01$, $\$ \$ \mathrm{p}<0.001$, rest value DCM-LBBB versus rest value DCM-NA (unpaired $t$ test).

A, peak late diastolic inflow; E, peak early diastolic inflow; ET, time from onset of aortic flow to A2; PAA, peak aortic acceleration. 

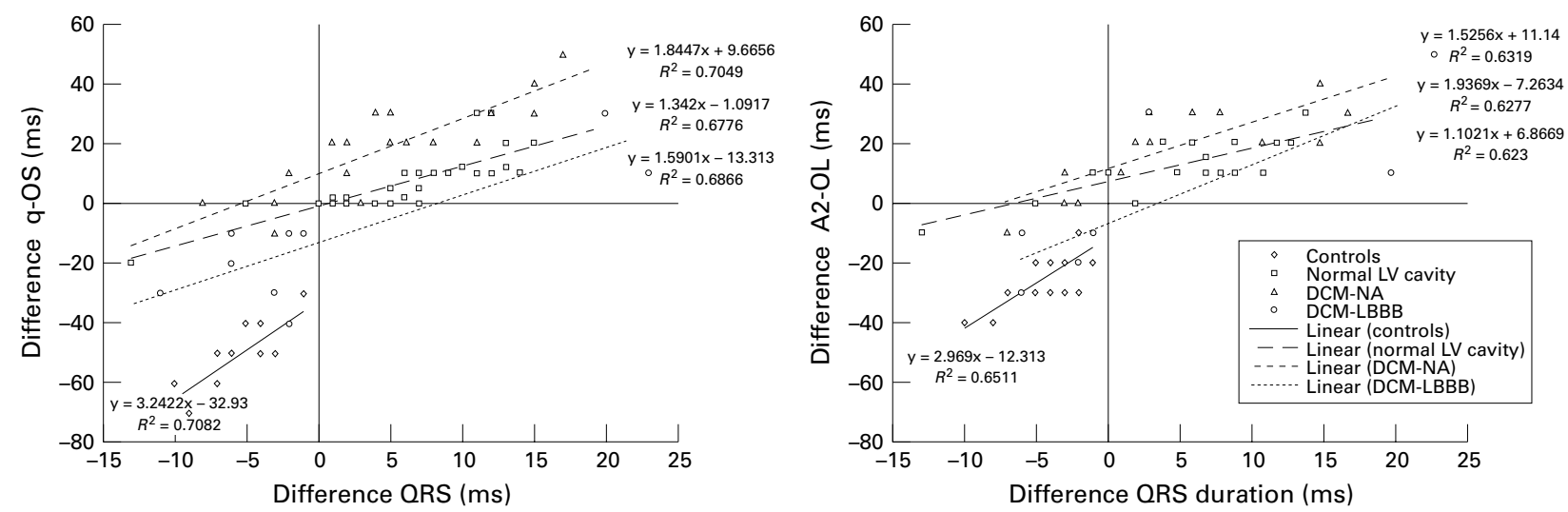

Figure 4 Correlation between the change in QRS duration and the time from the $Q$ wave to the onset of shortening ( $Q-O S$ ) in control and patient groups. In controls, $Q-O S$ shortened with dobutamine, even when the change in $Q R S$ duration was zero. This effect was attenuated in all patient groups. Similar relations were found between the change in QRS duration and the time from $A 2$ to the onset of lengthening (A2-OL) in all studied groups.

sooner after aortic valve closure, so that a consistently greater proportion of its overall shortening occurred during ejection indicating increased coordination. In individual control subjects, these altered time relations correlated with shortening of QRS duration, strongly suggesting that the resulting phase shift in long axis motion was, in part, the direct result of more rapid ventricular activation. This concurs with very early work by Wiggers, ${ }^{16}$ who suggested that normal left ventricular contraction relies on organised activation to develop coordinate tension in both circumferential and longitudinal fibres. There was, however, a second superimposed effect in that even when the change in QRS was zero the onset of shortening was $30 \mathrm{~ms}$ earlier with dobutamine. This latter effect is presumably a direct positive inotropic action of dobutamine, hastening the onset of contraction. Overall, therefore, dobutamine had a combined effect in the control group, both on activation and directly on the myocardium, so that the extent and velocity of long axis shortening increased and became more synchronous with ventricular ejection.

In patients with coronary disease, a positive inotropic effect of dobutamine could still be shown in terms of peak aortic acceleration, although its extent was attenuated in patients in whom ventricular cavity size was increased. The same applied to peak shortening velocity and, as in controls, changes in these two measurements correlated closely in individual patients. This suggests that in patients with coronary disease, peak long axis shortening velocity continues to reflect the direct positive inotropic effect of the drug in the same way as in control subjects. However, the time relations of long axis shortening were disturbed, in that the onset of shortening was delayed with respect to the Q wave of the ECG, and those of lengthening by a similar amount with respect to end ejection. The extent of the phase delay in long axis shortening correlated, as in controls, with the prolongation in QRS duration but the intercept of the relation did not differ from zero, showing loss of what we assume to be the direct inotropic effect on the timing of contraction. This phase delay had two effects. It led to the development of postejection shortening of the long axis, and it considerably reduced the extent to which overall long axis shortening was coupled to aortic ejection, representing loss of coordination. Patients with LBBB differed from those with normal activation in that the time course of long axis shortening was already delayed at rest and neither QRS duration nor the phase relations of long axis shortening were altered with dobutamine. In coronary disease, therefore, the overall long axis response to dobutamine was in part due to a varying degree of attenuation or loss of the positive inotropic effects but more significantly to incoordination resulting from altered ventricular activation, either present at rest or developing with stress.

It has been previously proposed that myocardial ischaemia is associated with disturbances of diastolic function. ${ }^{17}{ }^{18}$ In the present study, changes in transmitral flow velocities, commonly used to study diastolic function, were minor and non-specific during dobutamine stress and failed to distinguish control and patient groups. We were nevertheless able to identify two abnormalities that characteristically occurred during diastole. Postejection shortening appeared or increased during stress in all patients as a direct result of the phase shift of long axis shortening, but it was the direct consequence of altered activation. A second difference was that the normal increase in lengthening velocity with stress did not occur in any of the patient groups. Again, we hesitate to attribute this to a primary disturbance of diastolic function since, unlike peak shortening velocity, lengthening velocity correlated closely with the overall amplitude of shortening during systole. The relation between the two was similar at rest and during stress. This dependence of peak early diastolic lengthening velocity on systolic displacement makes a primary disturbance of relaxation an unlikely explanation and strongly suggests the operation of a simple elastic force activated by displacement of the atrioventricular ring towards the ventricular apex during ventricular systole. We have previously suggested that such forces may reside in the left atrium as well 
as in the left ventricular wall. ${ }^{19}$ We were thus unable to identify any primary disturbance of diastolic long axis function that may unequivocally be attributed to ischaemia or dobutamine stress.

Although much of the long axis response to dobutamine stress can be explained in terms of a combination of altered activation and direct positive inotropic effect, changes in long axis amplitude could not. The amplitude of long axis shortening consistently increased by 3-4 $\mathrm{mm}$ in controls but failed to do so in patients. In view of its major effect on lengthening velocity, further studies of stress induced amplitude changes are needed, both in patients with less severe CAD and in those who have been revascularised, to assess its potential reversibility.

\section{LIMITATIONS}

The study was not designed to use dobutamine stress echocardiography to diagnose CAD or to duplicate the effects of exercise, but rather to investigate electromechanical changes occurring with pharmacological stress. We did not, therefore, correlate our results with subjective wall motion scores. The end points reached were not uniform in that patients with normal cavity size all developed ECG changes of acute ischaemia, whereas in those with cavity dilatation, breathlessness was the dominant symptom. Since the condition of many of these patients with advanced left ventricular disease was potentially unstable during stress, the echocardiographic examination had to be performed expeditiously and was thus confined to recordings made from the apex. Furthermore, only septal long axis findings have been presented since the conducting system runs in the septal subendocardium. However, similar results were also found at the left and posterior left ventricular sites. Subgroup analysis showed that inclusion of patients with diabetes or hypertension or those taking an angiotensin converting enzyme inhibitor or $\beta$ blocker did not alter the results. The close correlation between shortening fraction and long axis excursion confirmed that the contraction pattern was symmetrical throughout the patients studied. A digitisation frequency of $100 \mathrm{~Hz}$ would have been adequate to describe changes in velocities of long axis motion but may have underestimated high values of peak aortic acceleration.

\section{CONCLUSION}

Our results confirm that ventricular activation is a labile process, showing it to change consistently during pharmacological stress. More significantly, these changes have predictable mechanical consequences in terms of the timing and coordination of ventricular wall motion. In normal subjects, shortening of the QRS complex results in an earlier onset and a more coordinate time course of long axis shortening, thus complementing the direct inotropic effects of dobutamine on the myocardium in enhancing ventricular performance. In patients with CAD, stress causes the QRS complex to broaden, with major loss of coordination of long axis function, even though the direct positive inotropic effect of dobutamine persists. Altered ventricular activation with consequent mechanical changes must therefore be considered as an integral part of the response of the left ventricular function to ischaemia. The effects of altered activation are particularly prominent during early diastole, where they must not be confused with primary manifestations of diastolic disease. Finally, our results shed light on factors that determine rates of change of mitral annulus position, which are increasingly being studied by tissue Doppler echocardiography. ${ }^{20}$ Peak systolic velocities, being closely related to early aortic acceleration, are likely to be a reliable measure of changes in the inotropic state of the left ventricle. Peak early diastolic velocity, often taken as a measure of "diastolic function", proved in normal subjects and patients with CAD both at rest and with stress to be simply a function of amplitude of the annulus displacement and thus of shortening fraction. It is on this simple basis rather than as any more profound index of intrinsic diastolic function that inclusion of peak early diastolic ring velocity is able to distinguish a normal from a so called pseudonormal $\mathrm{E}$ wave on the transmitral Doppler flow velocity trace. ${ }^{2122}$

Dr A Duncan is supported by The Clinical Research Committee of The Royal Brompton Hospital. We thank Dr Darrel Francis for helpful discussion.

1 Harvey W. An anatomical disputation concerning the movement of the heart and blood in living creatures. Oxford: Blackwell Scientific Publications, 1976:32-7.

2 Keith A. The functional anatomy of the heart. BMF 1918;i: 361-3.

3 Gibson DG, Prewitt TA, Brown DJ. Analysis of left ventricular wall motion during isovolumic relaxation and its relation to coronary artery disease. Br Heart f 1976;38: 1010-9.

4 Greenbaum RA, Ho SY, Gibson DG, et al. Left ventricular fibre architecture in man. Br Heart f 1981:45:248-63.

5 Alam M, Hoglund C, Thorstrand C, et al. Haemodynamic significance of the atrioventricular plane displacement in patients with coronary artery disease. Eur Heart f 1992;13: patients with $194-200$.

6 Henein MY, O'Sullivan CA, Davies SW, et al. Effects of acute coronary occlusion and previous ischaemic injury on left ventricular wall motion in humans. Heart 1997;77:33845

7 Xiao HB, Lee CH, Gibson DG. Effect of left bundle branch block on diastolic function in dilated cardiomyopathy. $\mathrm{Br}$ Heart f 1991;66:443-7.

8 Xiao HB, Gibson DG. Absent septal Q wave: a marker of the effects of abnormal activation pattern on left ventricular diastolic function. Br Heart $\mathcal{F}$ 1994;72:45-51.

9 Duncan AM, O'Sullivan CA, Gibson DG, et al. Electromechanical interrelations during dobutamine stress in normal subjects and patients with coronary disease: comparison of changes in activation and inotropic state. Heart 2001;85: 411-6.

10 Appleton CP, Hatle LK, Popp RL. Demonstration of restrictive physiology by Doppler echocardiography. $\mathcal{F} \mathrm{Am}$ Coll Cardiol 1988:11:757-68.

11 Henein MY, Gibson DG. Abnormal subendocardial function in restrictive left ventricular disease. Br Heart $\mathcal{F} 1994$; tion in restrict

12 Jones CJH, Raposo L, Gibson DG. Functional importance of the long axis dynamics of the human left ventricle. $\mathrm{Br}$ Heart f 1990;63:215-20

13 Pai RG, Bodenheimer MM, Pai SM, et al. Usefulness of systolic excursion of the mitral annulus as an index of left ventricular systolic function. Am F Cardiol 1991:15:222-4.

14 Gibson DG, Brown D. Measurement of instantaneous left ventricular dimension and filling rate in man, using echocardiography. Br Heart f 1973:35:1141-9.

15 Noble MIM, Trenchard D, Guz A. Left ventricular ejection in conscious dogs: measurement and significance of the maximum acceleration of blood from the left ventricle. Circ Res 1966;19:139-47.

16 Wiggers CJ. Are ventricular conduction changes important in the dynamics of ventricular contraction? $\mathrm{Am} \mathcal{F}$ Physiol $1926 ; 74: 12-30$ 
17 Labovitz AJ, Lewen MK, Kern M, et al. Evaluation of left ventricular systolic and diastolic dysfunction during transient myocardial ischaemia produced by angioplasty. $\mathscr{f}$ Am Coll Cardiol 1987;10:748-55.

18 El-Said ES, Roelandt JR, Fioretti PM, et al. Abnormal left ventricular early diastolic filling during dobutamine stress Doppler echocardiography is a sensitive indicator of significant coronary artery disease. $\mathcal{F}$ Am Coll Cardiol 1994; 24:1618-24.

19 Carr-White GS, Koh TW, Haxby E, et al. Mitral annulus energetics as determinants of left ventricular filling: quantification of atrioventricular interactions. Eur Heart f 2000:2 (suppl K):K61-8.
20 Garcia-Fernandez MA, Azevedo J, Moreno M, et al. Regional diastolic function in ischaemic heart disease using pulsed wave Doppler tissue imaging. Eur Heart 7 1999;20: 476-7

21 Farias CA, Rodriguez L, Garcia MJ, et al. Assessment of diastolic function by tissue Doppler echocardiography: comparison with standard transmitral and pulmonary venous flow. F Am Soc Echocardiogr 1999;12:609-17

22 Ohte N, Narita H, Hashimoto T, et al. Differentiation of abnormal relaxation pattern with aging from abnormal relaxation pattern with coronary artery disease in transmitral flow with the use of tissue Doppler imaging of the mitral annulus. I Am Soc Echocardiogr 1999;12:629-35.

\section{IMAGES IN CARDIOLOGY}

\section{Severe aneurysmal coronary artery disease}

A 59 year old white man was admitted to our hospital because of unstable angina. His past medical history was significant for an inferior myocardial infarction at the age of 52, hypertension, and hyperlipidaemia.

Coronary angiography was performed revealing severe aneurysmal coronary artery disease. The right anterior oblique projection (left) showed multiple aneurysms alternating with severe stenoses of the left anterior descending and circumflex coronary arteries. The left anterior oblique view (right) showed a large saccular aneurysm in the proximal segment of the right coronary artery as well as coronary ectasia and stenoses along the vessel. He underwent three vessel coronary artery bypass grafting with a left internal mammary artery to the left anterior descending artery and aneurysmectomy of the right coronary artery.

Coronary artery ectasia (CAE) is an uncommon angiographic finding. The description of

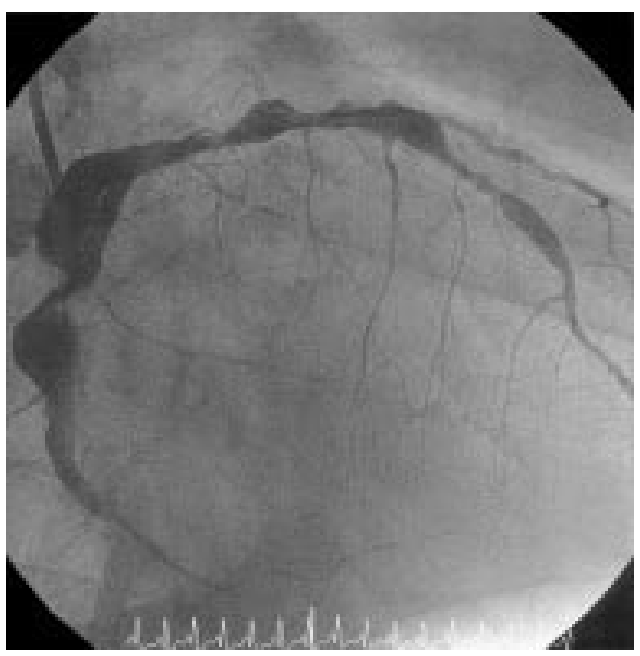

this disease in a syphilitic patient in the anatomical works of Giambattista Morgagni (1682-1771) is probably the earliest in the literature. In various other reports, CAE has been described either as an isolated congenital lesion or in association with Kawasaki's disease (mucocutaneous lymph node syndrome), scleroderma, polyarteritis nodosa, Ehlers-Danlos syndrome, hereditary haemorrhagic telangiectasia (Osler-Weber-Rendu), bacterial infection, and familial hypercholesterolaemia.

However, like in our patient, the vast majority of CAE is generally not a distinct anatomical or functional abnormality but an accompaniment or a variant of atherosclerotic coronary artery disease with which it usually coexists.

JAN S JÜRGENSEN MICHAEL SCHLEGL JÜRGEN HUG jan_steffen.juergensen@charite.de

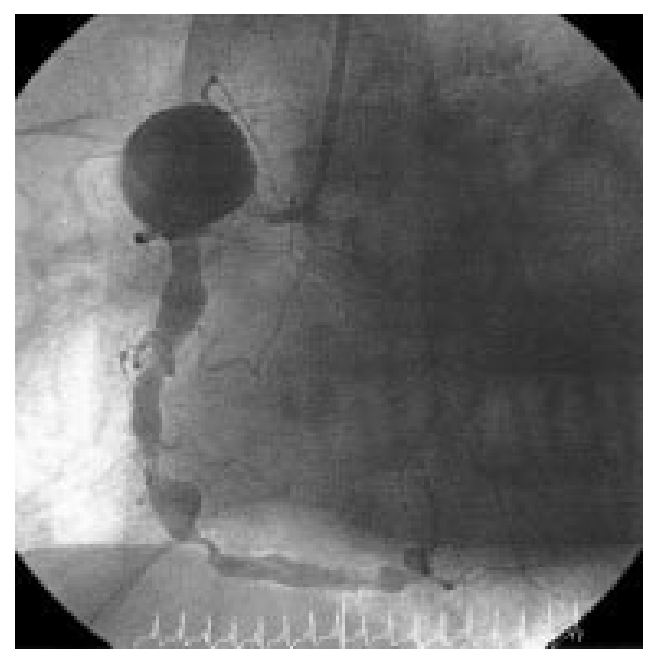

